\title{
Kidney regeneration and repair after transplantation
}

\author{
Marcella Franquesa ${ }^{\mathrm{a}, *}$, Maria Flaquer ${ }^{\mathrm{b}, *}$, Josep M. Cruzado ${ }^{\mathrm{b}}$, and \\ Josep M. Grinyób
}

\begin{abstract}
Purpose of review
To briefly show which are the mechanisms and cell types involved in kidney regeneration and describe some of the therapies currently under study in regenerative medicine for kidney transplantation.
\end{abstract}

\section{Recent findings}

The kidney contains cell progenitors that under specific circumstances have the ability to regenerate specific structures. Apart from the knowledge gained in the self-regenerative properties of the kidney, new concepts in regenerative medicine such as organ engineering and the use of mesenchymal stem cell-based therapies are currently the focus of attention in the field.

\section{Summary \\ Overall, kidney regeneration is a reality and the knowledge on how to control it will be one of the main scopes in the present and future.}

\section{Keywords}

hepatocyte growth factor, kidney, regenerative medicine, stem cells, transplantation

\section{INTRODUCTION}

Kidney transplantation is the best option for endstage renal disease patients. However, this is only a temporary solution and most of the patients develop interstitial fibrosis and tubular atrophy and eventually lose their grafts. A better knowledge on endogenous healing mechanisms for nonfibrotic wound healing and new therapies designed to increase the regenerative potential of the kidney might mean in a near future a longer graft survival or even no need for transplantation because of regeneration of native kidney.

In the context of kidney transplantation, the possibility to obtain biopsies regularly could provide a window of opportunity to detect stable grafts with initial signs of fibrosis, which could be used to foster regeneration before the onset of complete damage.

Until now, transplantation research has focused on the impact of the immunosuppressive drugs on the graft survival; however, these drugs might have a negative impact on the potential regeneration ability of the graft [1-3].

\section{ENDOGENOUS MECHANISMS OF RENAL REGENERATION}

The kidney has been classically considered unable to regenerate, in comparison with other organs such as the liver. Advances in developmental biology and in the knowledge of the balance between regeneration and fibrosis have brought major advances in the last years.

\section{Adult kidney stem cell niches}

When an adult tissue is damaged, the cell renewal process is crucial for its maintenance and, in certain organs, this is achieved by the presence of stem cells. These stem cell populations are housed in an in-vivo milieu, named a niche, which regulates stem cell survival, self-renewal, and differentiation. Stem cell niches are present in many adult organs and tissues [4]. In a normal environment, stem cells remain quiescent into the niche during long periods of time until they are activated by the requirement of new cells to maintain the tissue or because of tissue damage. When an insult happens, stem cells might be activated, exit the niche, proliferate, and

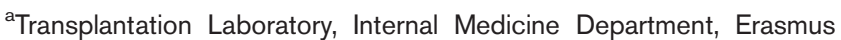
MC, Rotterdam, the Netherlands and ${ }^{\text {}}$ Nephrology Department, Hospital Univeristari de Bellvitge-IDIBELL, Barcelona, Spain

Correspondence to Marcella Franquesa, PO Box 2014, 3000 CA Rotterdam, The Netherlands. Tel: +31 107035 421; fax: +31 107 044 718; e-mail: m.franquesa@erasmusmc.nl

*This two authors contributed equally to this work.
}

Curr Opin Organ Transplant 2013, 18:191-196

DOI:10.1097/MOT.0b013e32835f0771 


\section{KEY POINTS}

- The kidney has intrinsic ability to regenerate after an injury.

- Several populations of stem cells with regenerative potential have been identified in the renal parenchyma.

- Regenerative medicine and organ engineering hold the potential to be the best alternatives to current treatments in transplantation.

- Mesenchymal stem cells and their derived microvesicles have been shown to protect from developing chronic injury.

- Other therapies such as hepatocyte growth factor or microvasculature regeneration enhances have already proven the regenerative potential in animal models.

differentiate into the needed cell types to replace the cell lost.

The kidney has a very complex structure and is composed of more than 20 cell types that function as a tissue unit rather than as individual cells, making harder its regeneration. These factors make it difficult to find a single renal stem cell able to differentiate into each renal cell type. Overcoming all these complications, some stem cell niches have been proposed in different renal compartments: the renal corpuscle, the nephron tubule, and the renal papilla.

\section{Glomerular populations (the renal corpuscle)}

Sagrinati et al. [5] identified, in human adult kidneys, a subset of parietal epithelial cells (PECs) in the Bowman's capsule that exhibit coexpression of the stem cell markers $\left(\mathrm{CD} 24^{+} \mathrm{CD} 133^{+}\right)$. CD24 is a surface molecule expressed in the developing human fetal kidneys [6] and it has been used to identify different types of human stem cells [7]. CD133 is also expressed in human fetal kidney (by around $50 \%$ of the cells) and only in $0.5-3 \%$ of the adult human kidney [8].

This $\mathrm{CD}_{24}{ }^{+} \mathrm{CD} 133^{+}$PEC population reveals self-renewal potential and a high cloning efficiency. The localization of this $\mathrm{CD} 24^{+} \mathrm{CD} 133^{+}$population is suitable for both podocyte and tubule regeneration. The infusion of $\mathrm{CD} 24^{+}$and $\mathrm{CD} 133^{+}$cells in SCID mice with acute renal failure resulted in the regeneration of tubular structures ameliorating the morphologic and functional kidney damage. A transitional cell population $\left(\mathrm{CD} 24^{+} \mathrm{CD} 133^{+} \mathrm{PDX}{ }^{+}\right)$ shares the features of either renal progenitors or podocytes and localizes between the urinary pole and the vascular pole [7]. More recently, Angelotti et al. [9"-] have demonstrated that this $\mathrm{CD} 24^{+}$ $\mathrm{CD} 133^{+}$progenitor population can be distinguished in distinct subpopulations from normal human kidneys based on the surface expression of vascular cell adhesion molecule 1 (CD106). Thus, it has been described in two subpopulations: $\mathrm{CD} 24^{+} \mathrm{CD} 133^{+}$ CD106 ${ }^{+}$which is localized at the urinary pole and $\mathrm{CD} 24^{+} \mathrm{CD} 133^{+} \mathrm{CD} 106^{-}$localized in the proximal tubule as well as in the distal convoluted tubule. The $\mathrm{CD} 24^{+} \mathrm{CD} 133^{+} \mathrm{CD} 106^{+}$subpopulation exhibits a high proliferative rate and can differentiate toward the podocyte as well as the tubular lineage. By contrast, the $\mathrm{CD} 24^{+} \mathrm{CD} 133^{+} \mathrm{CD} 106^{-}$subpopulation shows a lower proliferative capacity and solely differentiates into the tubular lineage. Both subpopulations, when injected into SCID mice with acute tubular injury, were able to engraft within the kidney, generate tubular cells, and improve renal function.

Bruno et al. [10"'] identified another glomerular population with stem cell features. They found that human glomeruli deprived of the Bowman's capsule contain a population of $\mathrm{CD} 133^{-} \mathrm{CD} 146^{+}$ cells that coexpress the typical mesenchymal stem cell markers (such as CD29, CD105, and CD73) and renal-specific stem cell markers (such as CD24 and Pax2). This population exhibits in-vitro self-renewal capability, clonogenicity, and multipotency. Moreover, when these cells are cultured in the appropriate culture conditions, they are able to differentiate into endothelial cells, epithelial cells expressing podocyte markers, and mesangial cells.

\section{Tubular populations}

The tubules of the kidney display a remarkable capacity for self-renewal on damage. Whether this regeneration is mediated by dedifferentiating surviving cells or stem cells has not been completely settled. On one hand, Humphreys et al. [11] described that nonlethally injured cells repopulate the kidney epithelium after injury in the absence of any specialized progenitor cell population. On the other hand, Lindgren et al. [12] detected a progenitor cell population in human kidney scattered through the proximal tubules which expressed the markers previously described for PECs, CD24 and CD133, and displays mixed features of renal progenitors and tubular cells. These tubular progenitors may proliferate and differentiate after tubular injury to replace dead cells. Moreover, it is well known that $\mathrm{CD} 24^{+} \mathrm{CD} 133^{+}$(either PECs or tubular populations) express cytokeratin 7, cytokeratin 19, BLC2 (antiapoptotic gene), MYOF (regenerationpromoting molecule), and vimentin. Although this 
tubular population has been identified, the identification of novel markers that may allow distinction between $\mathrm{CD}_{24}{ }^{+} \mathrm{CD} 133^{+}$renal progenitors of Bowman's capsule and CD $24^{+} \mathrm{CD} 133^{+}$tubular cells is still required, as has been performed for podocytes $\left(\mathrm{CD} 24^{+} \mathrm{CD} 133^{+} \mathrm{PDX}^{-}\right.$vs. $\left.\mathrm{CD} 24^{+} \mathrm{CD} 133^{+} \mathrm{PDX}^{+}\right)$.

Otherwise, according to findings by Patschan and Oliver, in which they described the migration of nestin-positive cells following acute kidney injury $[13,14]$, an overexpression of nestin in proximal tubules that may participate in the cell migration during renal repair has been recently observed [15].

\section{The renal papilla}

Oliver et al. determined, some years ago, another niche for adult kidney stem cells [14]. These authors detected a cell population in the renal papilla, which had a slow cycling rate. It was observed that during the repair phase of transient renal ischemia, these cells entered the cell cycle. More recently, these authors described that the SDF-1/CXCR4 axis is a critical regulator of papillary label-retaining cells activation following transient kidney injury and during organ repair [16].

\section{REGENERATIVE MEDICINE}

Cell therapies and organ engineering are two major lines of research in kidney regenerative medicine (Fig. 1). Advances in the field are fast growing and changing concepts.

\section{Organ engineering}

One of the research lines that has attracted much of the attention in the last years in kidney regeneration is the use of decellularized organs. Bioengineering custom-made organs, in which the cellular component is autologous and has an internal vascular network, will theoretically overcome the two major hurdles in transplantation: the shortage of organs and the toxicity deriving from lifelong immunosuppression.

This procedure is based on ex-vivo tissue regeneration using a donor scaffold by repopulating it with recipient-induced pluripotent stem cells (iPSCs) or embryonic stem cells. Although a few groups have been able to obtain a decellularized kidney scaffold [17], the success in recellularizing it has been rather scarce and limited to small vessels, arterioles, and glomeruli being repopulated by endothelial cells [18] and some tubular repopulation [19]. The structural complexity of the kidney, composed of more than 20 cell types, converts this into a difficult endeavor compared with the partial success obtained in heart, lung and liver $[20,21]$.

\section{Mesenchymal stem cells}

Mesenchymal stem cells are stromal cells that can be found and isolated from virtually every tissue. Because of interesting multilineage differentiation potential and their ability to migrate to the site of injury, they have been studied in the last 10 years as a therapeutic agent in kidney injury. Although initial studies observed migration of the injected cells to injured kidney and even tubular replacement [22], there is no solid evidence that those cells can regenerate damaged tissue (by differentiation or fusion) and truly give rise to kidney-specific cell populations [23].

Although some studies have suggested that about $2 \%$ of the injected MSCs engraft the injured kidney [24], a recent study proved rapid clearance

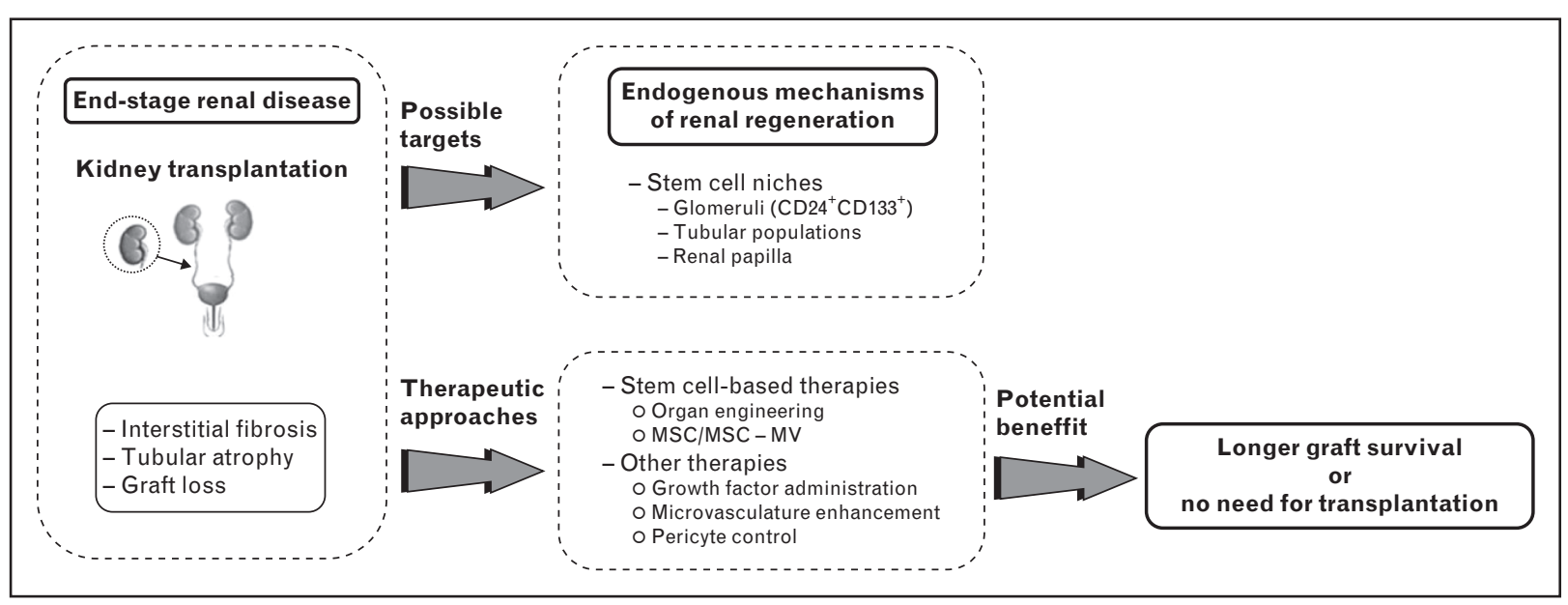

FIGURE 1. Scheme of the targets and therapeutic approaches in regenerative medicine. 
of the injected cells that get trapped in the lungs making impossible to detect any live injected cell after $24 \mathrm{~h}$ [25"']. In that respect, to overcome the fast clearance other injection routes (renal artery or femoral artery) have been developed to increase the chances of MSCs to get in touch with the kidney $[23,26]$. However, even when MSCs are injected systemically they exert a clear immunosuppressive effect that can lead to long-term reduction of interstitial fibrosis and tubular atrophy signs of chronic kidney damage [27"]. This effect is partially explained by the reduction in inflammatory infiltrate that mediates tissue scarring, and also by the ability of MSCs to shift the immunologic balance toward a more anti-inflammatory milieu (M2 macrophages, regulatory $\mathrm{T}$ cells, tolerogenic dendritic cells) [28]. However, the antiinflammatory actions per se are generally not sufficient to protect renal function and repair the damaged organ.

Overall, the consensus about the regenerative potential of MSCs is that they do not differentiate or fuse into any kidney cell type, MSCs will only reach the renal parenchyma when injected bypassing the lungs, and that their mechanism of action is through paracrine release of factors. Those factors are also found in the conditioned media, which when injected exert similar protective functions as MSCs, and one of the most plausible mechanism might be through released microvesicles.

\section{Microvesicles}

The use of MSC-derived microvesicles as a therapeutic agent to induce regeneration or at least to reduce kidney rejection is a new approach that evolved from the inconvenience of using whole replicating cells and might be the mechanistic explanation to solve the differential clearance/ effect observed with MSCs. Microvesicles are spontaneously released by many cell types including MSCs, and they can present surface receptors and contain biologically active proteins and lipids as well as mRNA or microRNA. Therefore, they have a potential role in the exchange of genetic material between cells [29"'].

In addition to their immunomodulatory properties (same as MSCs), they showed potential to activate proliferation in tubular epithelial cells in glycerol-induced acute kidney injury combined with severe combined immunodeficiency (SCID) mice model [30]. This effect was comparable with the one obtained with MSCs and was RNA dependent. Similar effect was observed in a model of renal ischemia/reperfusion injury, not only inhibiting acute injury but also preventing the development of chronic kidney disease [31].

\section{Other therapeutic approaches}

As research in the field is growing, other therapies and molecular mechanisms have been described (Fig. 1).

\section{Hepatocyte growth factor}

Hepatocyte growth factor (HGF) has been broadly studied because of its antifibrotic and proregenerative properties in kidney damage models. This short-lived growth factor presented some issues in the administration as a protein and it soon moved forward to an experimental gene therapy application to provide sustained expression. This has proved effective in acute ischemia/ reperfussion injury [32] and in chronic kidney damage after kidney transplantation [33]. One of the main mechanisms of action was the shift in the balance with TGF $\beta$ inhibiting pro-scarring factors, and it has been recently shown that when this therapy is applied together with a hematopoietic stem cell (HSC) mobilization factor (G-CSF), regeneration of the injured kidney is enhanced by the migration of bone-marrow-derived M2 macrophages to the site of injury [34"].

This regenerative effect induced by the migration of macrophages is in line with the effect observed by Jung et al. [35"] of acute kidney injury repair after injecting M2 macrophages overexpressing IL-10.

\section{Microvasculature and pericytes}

Chronic kidney disease is characterized by progressive loss of the renal microvasculature. Hence, it is proposed that revascularization might be a key process to restore kidney function and regenerate the damaged structure. There are many therapeutic approaches proposed to regenerate the microvasculature, such as vascular endothelial growth factor, angiopoietin-1 and endothelial progenitor cells (reviewed in [36]), HSC [37], MSCs [38], and the preservation of the pericytes.

Pericytes are cells of mesenchymal origin that manage and coordinate endothelial action and are also able to coordinate between endothelium and epithelium [39]. In response to injury, pericytes detach from the capillary walls and migrate into the interstitium, where they are activated contributing to fibrosis $\left[40^{-}\right]$. This detachment evidences the role of pericytes with regard to microvasculature. In contrary, blocking pericyte detachment not only results in a reduction in interstitial fibrosis but also prevents capillary rarefaction. Thus, strategies to modulate pericyte function in these fibrotic processes are therefore therapeutically attractive [41]. 


\section{CONCLUSION}

The kidney is a complex organ with a complex structure that, in contrast with what was believed, has the intrinsic capacity to regenerate. New knowledge on progenitor cells within the kidney, which in a steady state are responsible for correct kidney repair, and on their use as a therapeutic targets might be a key in the near future to reduce fibrotic scarring or even induce regeneration. However, future research will tell us whether these newly discovered progenitors are responsible for the regeneration mediated by endogenous signals or if they need external signaling (from infiltrating cells) to start the regenerative process. Regardless of the initial level of function, all transplanted kidneys suffer some ischemia and reperfusion injury followed by intrinsic regeneration. As biopsies are commonly obtained, the kidney transplant could provide valuable information about the regeneration of the human kidney.

Cell-based therapies are now being developed with promising results. MSCs seem to play a role in the reduction of damage, but their regenerative role still needs to be proved whether it is only a secondary effect to their immunoregulatory properties. However, their regenerative potential might be reduced because of their size after culturing. This problem might be bypassed by local injection of MSCs, the use of microvesicles, or new strategies to come. Organ engineering of the kidney is also moving fast and a full success in this field could be the final solution to all the problems related to organ transplantation. However, because of the complexity of the kidney in structure and cell types, a lot of research has to be done before we reach that point.

Other therapies are also currently studied among which we emphasize the use of HGF and microvasculature repairing mechanisms. Both represent important therapeutic approaches that have shown interesting outcomes, although their use might be in combination with other therapies.

Overall, kidney regeneration is a reality and the knowledge of how to control it will be one of the main scopes in the present and future.

\section{Acknowledgements}

None.

\section{Conflicts of interest}

There are no conflicts of interest.

Marcella Franquesa is a postdoctoral fellow funded by FP7 project RegenerAR. This work was funded by Ministerio Ciencia e Innovación-ISCIII (PI061073, PS09/01630) grants, by a European Union grant [SysKid project, Framework Programme 7 (FP7)], and by ISCIIIRETIC REDinREN/RD06/0016 grant.

\section{REFERENCES AND RECOMMENDED \\ READINC}

Papers of particular interest, published within the annual period of review, have been highlighted as:

- of special interest

- of outstanding interest

Additional references related to this topic can also be found in the Current

World Literature section in this issue (p. 244).

1. Yang CW, Lee SH, Lim SW, et al. Cyclosporine or fk506 decrease mature epidermal growth factor protein expression and renal tubular regeneration in rat kidneys with ischemia/reperfusion injury. Nephron 2002; 92:914-921.

2. Goncalves GM, Cenedeze MA, Feitoza $\mathrm{CQ}$, et al. The role of immunosuppressive drugs in aggravating renal ischemia and reperfusion injury. Transplant Proc 2007; 39:417-420.

3. Ysebaert DK, De Greef KE, Vercauteren SR, et al. Effect of immunosuppression on damage, leukocyte infiltration, and regeneration after severe warm ischemia/reperfusion renal injury. Kidney Int 2003; 64:864-873.

4. Krankel N, Spinetti G, Amadesi $S$, et al. Targeting stem cell niches and trafficking for cardiovascular therapy. Pharmacol Ther 2011; 129:62-81.

5. Sagrinati C, Netti GS, Mazzinghi B, et al. Isolation and characterization of multipotent progenitor cells from the bowman's capsule of adult human kidneys. J Am Soc Nephrol 2006; 17:2443-2456.

6. Ivanova L, Hiatt MJ, Yoder MC, et al. Ontogeny of CD24 in the human kidney. Kidney Int 2010; 77:1123-1131.

7. Romagnani P. Toward the identification of a 'Renopoietic system'? Stem Cells 2009; 27:2247-2253.

8. Bussolati B, Camussi G. New insights into the renal progenitor cells and kidney diseases by studying CD133. In: Corbeil D, editor. Prominin-1 (cd133): new insights on stem \& cancer cell biology. New York: Springer Science + Business Media; 2013.

9. Angelotti $\mathrm{ML}$, Ronconi $\mathrm{E}$, Ballerini $\mathrm{L}$, et al. Characterization of renal

- progenitors committed toward tubular lineage and their regenerative potential in renal tubular injury. Stem Cells 2012; 30:1714-1725.

This study demonstrates that $\mathrm{CD} 24^{+} \mathrm{CD} 133^{+}$renal progenitors can be distinguished into two progenitor subpopulations based on the CD106 marker. Whereas $\mathrm{CD}_{2} 4^{+} \mathrm{CD} 133^{+} \mathrm{CD} 106^{+}$shows a high proliferative rate and can differentiate toward the podocyte as well as tubular lineage, CD24 ${ }^{+}$CD $133^{+}$ CD106 ${ }^{-}$shows a lower proliferative capacity representing tubular committed progenitors and exert regenerative potential for injured tubular tissue.

10. Bruno S, Camussi G. Isolation and characterization of resident mesenchymal - stem cells in human glomeruli. Methods Mol Biol 2012; 879:367-380.

This study presents experimental procedures used for isolation of a population of CD133 ${ }^{-}$CD $146^{+}$cells that coexpress MSC markers (CD29, CD105, and CD73) and renal-specific stem cell markers (CD24 and Pax2), which is able to differentiate into endothelial cells, epithelial cells expressing podocyte markers and mesangial cells, and may have a role in the physiological cell turnover in response to renal injury.

11. Humphreys BD, Czerniak $S$, DiRocco DP, et al. Repair of injured proximal tubule does not involve specialized progenitors. Proc Natl Acad Sci USA $2011 ; 108: 9226-9231$.

12. Lindgren $D$, Bostrom $A K$, Nilsson $K$, et al. Isolation and characterization of progenitor-like cells from human renal proximal tubules. Am J Pathol 2011; 178:828-837.

13. Patschan $D$, Michurina $T$, Shi HK, et al. Normal distribution and medullary-tocortical shift of nestin-expressing cells in acute renal ischemia. Kidney Int 2007; 71:744-754.

14. Oliver JA, Maarouf $\mathrm{O}$, Cheema $\mathrm{FH}$, et al. The renal papilla is a niche for adult kidney stem cells. J Clin Invest 2004; 114:795-804.

15. Wen D, Ni L, You L, et al. Upregulation of nestin in proximal tubules may participate in cell migration during renal repair. Am J Physiol Renal Physiol 2012; 303:F1534-F1544.

16. Oliver JA, Maarouf $\mathrm{O}$, Cheema FH, et al. SDF-1 activates papillary labelretaining cells during kidney repair from injury. Am J Physiol Renal Physiol 2012; 302:F1362-F1373.

17. Sullivan DC, Mirmalek-Sani SH, Deegan DB, et al. Decellularization methods of porcine kidneys for whole organ engineering using a high-throughput system. Biomaterials 2012; 33:7756-7764.

18. Ross EA, Abrahamson DR, St John $P$, et al. Mouse stem cells seeded into decellularized rat kidney scaffolds endothelialize and remodel basement membranes. Organogenesis 2012; 8:49-55.

19. Nakayama $\mathrm{KH}$, Batchelder $\mathrm{CA}$, Lee $\mathrm{Cl}$, et al. Renal tissue engineering with decellularized rhesus monkey kidneys: age-related differences. Tissue Eng Part A 2011; 17:2891-2901.

20. Song JJ, Ott HC. Organ engineering based on decellularized matrix scaffolds. Trends Mol Med 2011; 17:424-432. 
21. Orlando $G$, Baptista $P$, Birchall $M$, et al. Regenerative medicine as applied to solid organ transplantation: Current status and future challenges. Transpl Int $2011 ; 24: 223-232$

22. Morigi M, Imberti $B$, Zoja $C$, et al. Mesenchymal stem cells are renotropic, helping to repair the kidney and improve function in acute renal failure. J Am Soc Nephrol 2004; 15:1794-1804.

23. Togel FE, Westenfelder C. Kidney protection and regeneration following acute injury: progress through stem cell therapy. Am J Kidney Dis 2012; 60:1012-1022.

24. Herrera MB, Bussolati $B$, Bruno $S$, et al. Exogenous mesenchymal stem cells localize to the kidney by means of CD44 following acute tubular injury. Kidney Int 2007; 72:430-441.

25. Eggenhofer E, Benseler V, Kroemer A, et al. Mesenchymal stem cells are

- short-lived and do not migrate beyond the lungs after intravenous infusion. Front Immunol 2012; 3:297.

In this interesting article about the fate of intravenously injected MSCs, the authors prove lung entrapment and fast death of the cells. This brings the MSC therapies to a new paradigm as the effect of the cells is observed long-term after injection.

26. Zonta $S$, De Martino $M$, Bedino $G$, et al. Which is the most suitable and effective route of administration for mesenchymal stem cell-based immunomodulation therapy in experimental kidney transplantation: endovenous or arterial? Transplant Proc 2010; 42:1336-1340.

27. Franquesa $M$, Herrero $E$, Torras $J$, et al. Mesenchymal stem cell therapy prevents interstitial fibrosis and tubular atrophy in a rat kidney allograft model. Stem Cells Dev 2012; 21:3125-3135.

In this study, the authors use for the first time MSC therapeutic injection to treat chronic kidney injury after transplantation.

28. Franquesa M, Hoogduijn MJ, Baan CC. The impact of mesenchymal stem cell therapy in transplant rejection and tolerance. Curr Opin Organ Transplant $2012 ; 17: 355-361$.

29. Biancone L, Bruno S, Deregibus $M C$, et al. Therapeutic potential of

n. mesenchymal stem cell-derived microvesicles. Nephrol Dial Transplant 2012; 27:3037-3042.

An interesting review about the potential of microvesicles to prevent organ injury. Mechanisms involved are discussed.

30. Bruno S, Grange C, Deregibus MC, et al. Mesenchymal stem cell-derived microvesicles protect against acute tubular injury. J Am Soc Nephrol 2009; 20:1053-1067.
31. Gatti S, Bruno S, Deregibus MC, et al. Microvesicles derived from human adult mesenchymal stem cells protect against ischaemia-reperfusioninduced acute and chronic kidney injury. Nephrol Dial Transplant 2011; 26:1474-1483

32. Franquesa $M$, Alperovich $G$, Herrero-Fresneda I, et al. Direct electrotransfer of hHGF gene into kidney ameliorates ischemic acute renal failure. Gene Ther $2005 ; 12: 1551-1558$

33. Herrero-Fresneda I, Torras J, Franquesa M, et al. HGF gene therapy attenuates renal allograft scarring by preventing the profibrotic inflammatoryinduced mechanisms. Kidney Int 2006; 70:265-274.

34. Flaquer $M$, Franquesa $M$, Vidal $A$, et al. Hepatocyte growth factor gene

- therapy enhances infiltration of macrophages and may induce kidney repair in $d b / d b$ mice as a model of diabetes. Diabetologia 2012; 55:20592068.

In this study, the authors describe a combined therapy with HGF and G-CSF that induces pro-regenerative macrophage infiltration in the kidney.

35. Jung $M$, Sola A, Hughes J, et al. Infusion of IL-10-expressing cells protects

- against renal ischemia through induction of lipocalin-2. Kidney Int 2012; 81:969-982.

A very interesting approach for kidney damage protection by injection of pro-regenerative macrophages.

36. Long DA, Norman JT, Fine LG. Restoring the renal microvasculature to treat chronic kidney disease. Nat Rev Nephrol 2012; 8:244-250.

37. Odorfer KI, Egerbacher M, Unger NJ, et al. Hematopoietic bone marrow cells participate in endothelial, but not epithelial or mesenchymal cell renewal in adult rats. J Cell Mol Med 2011; 15:2232-2244.

38. Eirin A, Zhu XY, Krier JD, et al. Adipose tissue-derived mesenchymal stem cells improve revascularization outcomes to restore renal function in swine atherosclerotic renal artery stenosis. Stem Cells 2012; 30:1030-1041.

39. Schrimpf C, Duffield JS. Mechanisms of fibrosis: the role of the pericyte. Curr Opin Nephrol Hypertens 2011; 20:297-305.

40. Lin SL, Chang FC, Schrimpf $C$, et al. Targeting endothelium-pericyte cross

- talk by inhibiting VEGF receptor signaling attenuates kidney microvascular rarefaction and fibrosis. Am J Pathol 2011; 178:911-923.

Demonstration of the potential of targeting pericytes as a mechanism of renal damage control.

41. Smith SW, Schrimpf C, Parekh DJ, et al. A novel therapeutic target in interstitial fibrosis. Histol Histopathol 2012; 27:1503-1514. 